\title{
Visual marking beside the mark: Prioritizing selection by abrupt onsets
}

\author{
MIEKE DONK and JAN THEEUWES \\ Vrije Universiteit, Amsterdam, The Netherlands
}

\begin{abstract}
In a standard visual marking experiment, observers are presented with a display containing one set of elements (old elements) followed after a certain time interval by a second set of elements (new elements). The task of observers is to search for a target among the new elements. Typically, the time to find the target depends only on the number of new elements in the display and not on the number of old elements, showing that observers search only among the new elements. This effect of prioritizing new elements over old elements is explained in terms of top-down inhibition of old objects-that is, visual marking (Watson \& Humphreys, 1997). The present study addressed whether this prioritizing is in fact mediated by top-down inhibition of old objects, as suggested by Watson and Humphreys (1997), or whether it is mediated by the abrupt onsets of the newly presented elements (Yantis \& Jonides, 1984). In three experiments, the presentations of the old and new elements were or were not accompanied by a luminance change. The results showed that if new elements were equiluminant with the background, no visual marking occurred, suggesting that new elements must have a luminance onset in order to be prioritized over old elements. Implications for current theories on visual selection are discussed.
\end{abstract}

Recently, Watson and Humphreys (1997) proposed a new mechanism for prioritizing the selection of new objects over objects that have already been present longer in the visual field, which they referred to as "visual marking" (see also Theeuwes, Kramer, \& Atchley, 1998; Watson \& Humphreys, 1998). According to Watson and Humphreys (1997), visual marking is the top-down inhibition of old objects that occurs because observers inhibit locations of old objects so that these objects no longer compete as strongly for selection. They have proposed that visual marking is a resource-limited intentional process that can be voluntarily switched on and off (Watson \& Humphreys, 1997). Their basic experiment consisted of three conditions. In the feature condition, observers searched for a blue $\mathrm{H}$ among blue As. In the conjunction condition, observers searched for a blue $\mathrm{H}$ among blue As and green Hs. In the gap condition, observers were presented with a display containing a number of green Hs (old elements) followed after a 1,000-msec interval by the addition of a number of blue As and possibly a blue $\mathrm{H}$ (new elements). The task of observers was to search for the blue $\mathrm{H}$ that was, if present, known to be presented among the new elements. Typically, reaction time (RT) in the feature condition was found to be independent of the number of elements in the display, whereas RT in the conjunction condition increased linearly with the number of elements, with a 2:1 slope ratio between target-absent

We thank A. Gellatly, G. W. Humphreys, and A. F. Kramer for comments on an earlier version of the article. Correspondence should be addressed to M. Donk, Department of Cognitive Psychology, Vrije Universiteit, Van Der Boechorststraat 1, 1081 BT Amsterdam, The Netherlands (e-mail: w.donk@psy.vu.nl). and target-present trials. More important was the finding that search performance in the gap condition was similar to that in the feature condition and dissimilar to that in the conjunction condition. The observation that search performance in the gap condition was equivalent to that in the feature condition was taken as evidence that the selection of new elements was prioritized. Watson and Humphreys (1997) concluded that the prioritized selection of new elements was mediated by top-down inhibition of the locations of the old elements.

Apart from demonstrating the basic phenomenon, Watson and Humphreys (1997) reported an impressive series of experiments in which they ruled out a variety of alternative theoretical explanations for their findings. To exclude an account that assumes that new objects are directly prioritized for selection through their abrupt onsets ${ }^{1}$ (Yantis \& Jonides, 1984), Watson and Humphreys (1997) performed an experiment (Experiment 5) in which in the gap condition the old elements underwent a luminance decrement upon the appearance of the new elements. The new elements were presented with a luminance onset. Watson and Humphreys (1997) argued that if prioritizing is mediated by abrupt onsets instead of visual marking, and if onsets dominate offsets in visual selection, as suggested by a study of Yantis and Johnson (1990), local luminance decrements in the old elements concurrently with the onsets of the new elements should not change the basic finding of prioritized selection of the new elements. The important result was that search performance in the gap condition was similar to that in the conjunction condition and dissimilar to that in the feature condition, suggesting that prioritizing did not occur. On the basis of these results, Watson and Humphreys (1997) discarded an account as- 
serting that prioritized selection of new elements, as found in previous experiments, was mediated by the abrupt onsets of the new elements. Instead, they argued that prioritizing of new elements occurred through top-down inhibition of old objects-that is, visual marking. Although such an account may be valid, the results of Experiment 5 do not necessarily imply that abrupt onsets did not play a role. If onsets do not dominate offsets in visual selection, in Experiment 5 observers might have been unable to neglect the offsets, resulting in a redeployment of attentional resources over both old and new elements. Indeed, Watson and Humphreys (1997) argued that the results of Yantis and Johnson suggesting that onsets dominate offsets might be refuted on the basis of the fact that their "new object onset stimuli produced a greater luminance change than the offsets" (Watson \& Humphreys, 1997, p. 107). Furthermore, there are various studies suggesting that offsets do at least modulate the extent to which onsets receive prioritized selection. For example, Miller (1989) showed that when observers searched for a target letter among nontarget letters, onset targets did not necessarily receive priority over offset elements. Miller concluded that both onset and offset transients have power to achieve prioritized selection. Evidence that offsets might be just as powerful as onsets in accomplishing prioritization was provided by Theeuwes (1991), who showed that if observers searched for a target letter among three nontarget letters, both a peripheral onset and a peripheral offset affected performance equally. In fact, visual offsets were found to be as effective as visual onsets in accomplishing prioritized selection. Recently, Martin-Emerson and Kramer (1997) performed three experiments in which they manipulated the ratio of offset to onset stimuli in the display as well as the ratio of offset to onset segments between the stimulus types. The results indicated that both manipulations affected search performance. MartinEmerson and Kramer concluded that offset transients modulate the extent to which sudden onsets receive prioritized selection. On the basis of these results, one might argue that it is premature to discard an account in terms of abrupt onsets for the finding of prioritizing new objects over old ones. The aim of the present study was to determine whether prioritizing new objects over old ones is due to the abrupt onsets of the new objects.

To test the hypothesis regarding whether or not prioritizing of new objects is mediated by abrupt onsets, three experiments were executed. In these experiments, observers searched for a green $\mathrm{H}$ among a variable number of other green letters. As in the gap condition of Watson and Humphreys (1997), a time interval was implemented between the presentation of old and new elements. The number of old elements $(5,10$, or 15 elements) and the number of new elements $(5,10$, or 15 elements) were orthogonally manipulated. The present paradigm deviates somewhat from the paradigm used by Watson and Humphreys (1997) but is identical to the one used by Theeuwes et al. (1998). The paradigm of Theeuwes et al. allows one to demonstrate the existence of a possible gap effect with- out the presence of a color confound, which might have been present in the original study of Watson and Humphreys (Theeuwes et al., 1998). Furthermore, in contrast to the paradigm used by Watson and Humphreys (1997), the present paradigm allows an independent manipulation of the number of elements before and after the gap, permitting a direct comparison between the effect of the number of old elements and that of the number of new elements on search performance. In three experiments, the presentations of the old and new elements were or were not accompanied by a luminance change. In Experiment 1, both old and new elements were equiluminant with the background so that there were no onsets in either set. In Experiment 2, the new elements were equiluminant with the background, whereas the old elements were not, yielding an onset of the old elements only. Finally, in Experiment 3 , the old elements were equiluminant with the background, whereas the new elements were not, providing an onset of the new elements.

\section{EXPERIMENT 1}

The aim of Experiment 1 was to investigate whether or not prioritizing selection of new elements occurs if both old and new elements are equiluminant with the background. If observers prioritize new elements over old elements by means of top-down inhibition of old elements (Watson \& Humphreys, 1997), it is expected that the number of old elements does not have any effect on the time to find the target. Search performance should depend only on the number of new elements in the display. Alternatively, if observers prioritize new elements due to the abrupt onsets of the new elements, search performance is predicted to depend on the number of new elements as well as the number of old elements.

\section{Method}

Participants. Five male and 3 female participants aged 21 to 36 years took part in the present experiment. Each participant had normal or corrected-to-normal vision.

Apparatus. A Celeron 400-MHz/128 Kb PC controlled the timing of events, the generation of the stimuli, and the recording of the responses. Stimuli were presented on a 19-in. Multiscan color monitor (with a ATI Rage 4-Mb card). The "z" key and the "/" key of the computer keyboard were utilized as response buttons. Participants were tested in a sound-isolated dimly lit room. They were seated at a distance of $95 \mathrm{~cm}$ from the computer monitor with their heads fixed in a head/chin rest.

Task and Stimuli. The task was identical to that of Theeuwes et al. (1998). Participants had to indicate the presence or absence of a target element among a variable number of nontarget elements. The target was always a capital letter $\mathrm{H}$, and the nontargets were capital letters randomly drawn (without replacement) from a subset of letters (A, B, C, E, F, G, J, K, M, N, P, R, S, T, U, V). In each trial, 5,10 , or 15 letters (old elements) were presented followed after $1,000 \mathrm{msec}$ by the addition of another 5,10 , or 15 letters (new elements). In $50 \%$ of the trials, the target was present and was presented among the new elements only. Targets and nontargets were green (CIE $x, y$ chromaticity coordinates of $.289, .605 ; 16.8 \mathrm{~cd} / \mathrm{m}^{2}$ ) and equiluminant with the gray background (CIE $x, y$ chromaticity coordinates of $.281, .327 ; 16.6 \mathrm{~cd} / \mathrm{m}^{2}$ ) as determined by a flicker fu- 
sion test (Ives, 1912) carried out at fixation. ${ }^{2}$ The letters, subtending a visual angle of $0.7^{\circ} \times 0.9^{\circ}$ at an observation distance of $95 \mathrm{~cm}$, were randomly positioned within a stimulus field of $14.9^{\circ} \times 10.7^{\circ}$ of visual angle.

Design and Procedure. A within-subjects design was used. Each participant completed two blocks of 216 trials. Each block was preceded by 20 practice trials. Number of old elements, number of new elements, and target presence were randomly varied within blocks of trials. There were three levels of number of old elements $(5,10$, and 15 elements), three levels of number of new elements $(5,10$, and 15 elements), and two levels of target presence (target present and target absent). Each trial started with the presentation of a tone, immediately followed by the presentation of a white fixation cross in the middle of the screen. After 1,500 msec, 5, 10, or 15 letters (old elements) were presented for $1,000 \mathrm{msec}$, after which 5,10 , or 15 letters (new elements) were added to the display. The display remained on until the participant responded, with a maximum of $6,000 \mathrm{msec}$. The fixation cross remained on throughout each trial. Participants were instructed to remain fixated until the new elements came on. Furthermore, they were explicitly told that if the target would be present, it would occur among the new elements. Half of the participants pressed the " $\mathrm{z}$ " key when the target " $\mathrm{H}$ " was present and pressed the "/" key when it was absent. This assignment was reversed for the other half of participants. Participants received feedback about their performance (in terms of RT and error rates) after each block of 50 trials.

\section{Results}

Figure 1 depicts the mean correct RT as a function of the number of old elements and the number of new elements separately for target-present and target-absent trials. Generally, RT was larger for target-absent trials than for target-present trials $[F(1,7)=110.05, p<.001]$.
For target-present trials, RT increased as a function of the number of old elements $[F(2,14)=7.58, p<.006]$ and the number of new elements $[F(2,14)=15.42, p<.001]$. There was no interaction between the number of old elements and the number of new elements $[F(4,28)=2.63$, $p>$.05].

For target-absent trials, RT also increased as a function of the number of old elements $[F(2,14)=33.35, p<$ $.001]$ and the number of new elements $[F(2,14)=90.91$, $p<.001]$. In addition, there was a significant interaction between the number of old elements and the number of new elements $[F(4,28)=4.14, p<.009]$, showing that the effects of the number of old and new elements decreased if the total number of elements increased. This finding suggests that observers utilized an upper time limit in finishing the self-terminating search process.

To investigate whether the speed of search was equal through the old elements and the new elements, best fitting lines were determined for the functions relating RT to the number of old elements and those relating RT to the number of new elements separately for each participant. Table 1 shows the mean search slopes and intercepts. Generally, search slopes were smaller for target-present trials than for target-absent trials $[F(1,7)=48.86, p<.001]$. For target-present trials, there was no difference between the search slope of the function relating RT to the number of old elements and that of the function relating RT to the number of new elements $[F(1,7)=1.44, p>.05]$. For target-absent trials, there was also no difference between the search slope of the function relating RT to the
Target Present

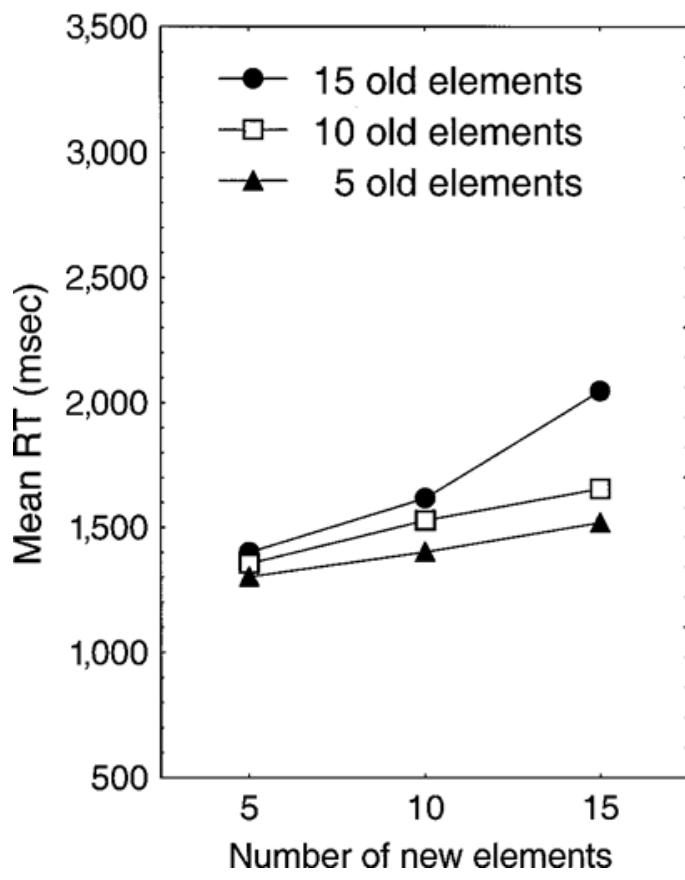

Target Absent

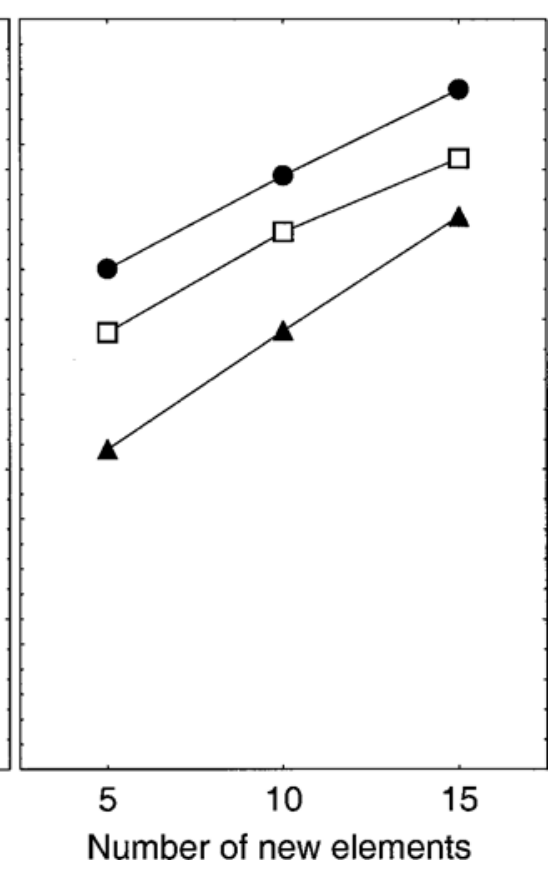

Figure 1. Correct mean reaction time (RT) as a function of the number of new and old elements separately for target-present and target-absent trials in Experiment 1. 
Table 1

Mean Intercepts, Search Slopes, and Absent/Present Ratios Separately for the Functions Relating Reaction Time to the Number of Old Elements $(\mathrm{OE})$ and Those Relating Reaction Time to the Number of New Elements (NE) in Experiments 1, 2, and 3

\begin{tabular}{|c|c|c|c|c|c|c|}
\hline & \multicolumn{6}{|c|}{ Experiment } \\
\hline & \multicolumn{2}{|c|}{1} & \multicolumn{2}{|c|}{2} & \multicolumn{2}{|c|}{3} \\
\hline & $\mathrm{OE}$ & NE & $\mathrm{OE}$ & NE & $\mathrm{OE}$ & $\mathrm{NE}$ \\
\hline \multicolumn{7}{|l|}{ Intercept } \\
\hline Present & 1,259 & 1,153 & 1,046 & 962 & 810 & 511 \\
\hline Absent & 1,959 & 1,793 & 1,805 & 1,791 & 1,123 & 474 \\
\hline \multicolumn{7}{|l|}{ Slope } \\
\hline Present & 27.7 & 37.9 & 30.8 & 39.2 & 2.7 & 33.1 \\
\hline Absent & 61.9 & 78.3 & 63.8 & 65.2 & 19.2 & 84.4 \\
\hline Absent/present ratio & 2.2 & 2.1 & 2.1 & 1.7 & 7.1 & 2.5 \\
\hline
\end{tabular}

number of old elements and that of the function relating RT to the number of new elements $[F(1,7)=2.63, p>.05]$. The results with respect to the intercepts of the functions were very similar: Intercepts were smaller for target-present than for target-absent trials $[F(1,7)=82.74, p<.001]$. For target-present trials, there was no difference between the intercept of the function relating RT to the number of old elements and that of the function relating RT to the number of new elements $[F(1,7)=1.53, p>.05]$. For the target-absent trials, there was also no difference between the intercept of the function relating RT to the number of old elements and that of the function relating RT to the number of new elements $[F(1,7)=2.71, p>.05]$.

Table 2 shows the mean percentages of errors. Overall, participants made $8.7 \%$ errors. Participants more often falsely reported the target to be absent when in fact the target was present than they falsely reported the target to be present when in fact the target was absent $[F(1,7)=$ $61.62, p<.001]$, showing that participants were more inclined to respond "target absent" than "target present." More important was the finding that error rates were affected neither by the number of old elements $[F(2,14)=$ $0.69]$ nor by the number of new elements $[F(2,14)=0.21]$.

\section{Discussion}

Experiment 1 shows that search performance depends on both the number of new elements and the number of old elements. This result is not compatible with the idea that the selection of new elements is prioritized by topdown inhibition of old objects, since then one would expect performance to be dependent only on the number of new elements (Watson \& Humpheys, 1997). Instead, in the present experiment no prioritizing occurred at all: Old and new elements were equally likely to be selected, as is evident from the increasing search functions for both old and new elements. Furthermore, there was no difference in search rates for old relative to new elements. Given the present finding, it seems unlikely that previous results suggesting the existence of visual marking (e.g., Theeuwes et al., 1998; Watson \& Humphreys, 1997, 1998) were actually due to inhibition of the initial set of elements. Instead, prioritizing the selection of new objects might have been related to the multiple onsets of the new elements. Alternatively, it might be argued that the absence of any prioritizing of new elements in the present Experiment 1 was due to the lack of onset of the old elements. Possibly in order to obtain top-down inhibition of old elements, it may be necessary that these old objects are initially presented with an onset. In other words, the initial equiluminant presentation of the old objects may have prevented top-down inhibition. Thus observers may only be able to exclude the initial nontargets from search by top-down inhibition if these elements are onset stimuli. This alternative was tested in Experiment 2.

\section{EXPERIMENT 2}

Experiment 1 established that no prioritizing occurs when both old and new elements are equiluminant with the background. The aim of Experiment 2 was to investigate whether the absence of prioritizing in Experiment 1 might have been related to the absence of onset of the old elements. Experiment 2 is basically a replication of Experiment 1 except that only the new elements were equiluminant with the background. The old elements were not equiluminant with the background, yielding an onset of the old elements only.

\section{Method}

Participants. Four male and 4 female participants aged 21 to 25 years took part in the present experiment. Each participant had normal or corrected-to-normal vision.

Apparatus. The apparatus was identical to that used in Experiment 1 .

Task and Stimuli. Task and stimuli were the same as in Experiment 1 except that the luminance of the background was lower upon appearance of the first set of elements (old elements), yielding an onset of the old elements (background: CIE $x, y$ chromaticity coordinates of $.281, .327,3.8 \mathrm{~cd} / \mathrm{m}^{2}$ ). The luminance of the background was gradually increased $732 \mathrm{msec}$ after presentation of the old elements (in 15 steps of $32 \mathrm{msec}$ each) up until the luminance of the elements during a period of $480 \mathrm{msec}$. Upon presentation of the sec-

Table 2

Mean Percentages of Errors in Experiment 1

\begin{tabular}{|c|c|c|c|c|c|c|c|c|c|}
\hline & \multicolumn{9}{|c|}{ No. Old Elements } \\
\hline & \multicolumn{3}{|c|}{$\begin{array}{c}5 \\
\text { No. New Elements } \\
\end{array}$} & \multicolumn{3}{|c|}{$\begin{array}{c}10 \\
\text { No. New Elements } \\
\end{array}$} & \multicolumn{3}{|c|}{$\begin{array}{c}15 \\
\text { No. New Elements }\end{array}$} \\
\hline & 5 & 10 & 15 & 5 & 10 & 15 & 5 & 10 & 15 \\
\hline Target present & 15 & 14 & 14 & 13 & 18 & 14 & 13 & 20 & 18 \\
\hline Target absent & 3 & 1 & 2 & 2 & 1 & 2 & 3 & 2 & 2 \\
\hline
\end{tabular}


ond set of elements, the background luminance was identical to that of both sets of elements (background: CIE $x, y$ chromaticity coordinates of $.281, .327,16.6 \mathrm{~cd} / \mathrm{m}^{2}$; elements: CIE $x, y$ chromaticity coordinates of $.289, .605,16.8 \mathrm{~cd} / \mathrm{m}^{2}$ ). The total time between the presentation of the old and new elements was $1,244 \mathrm{msec}$, an interval long enough to allow the development of visual marking.

Design and Procedure. The design and procedure were identical to those used in Experiment 1.

\section{Results}

Figure 2 depicts the mean correct RT as a function of the number of old elements and the number of new elements separately for target-present and target-absent trials. Generally, RT was larger for target-absent trials than for target-present trials $[F(1,7)=178.51, p<.001]$.

For target-present trials, RT increased as a function of the number of old elements $[F(2,14)=18.70, p<.001]$ and as a function of the number of new elements $[F(2,14)=$ $31.22, p<.001]$. There was no interaction between the number of old elements and the number of new elements $[F(4,28)=1.08, p>.05]$.

For target-absent trials, RT also increased as a function of the number of old elements $[F(2,14)=97.27, p<$ $.001]$ and as a function of the number of new elements $[F(2,14)=51.49, p<.001]$. In addition, there was a significant interaction between the number of old elements and the number of new elements $[F(4,28)=9.35, p<.001]$, showing that the effects of the number of old and new elements decreased if the total number of elements increased. Again, this finding suggests that observers utilized an upper time limit in finishing the self-terminating search process.
To investigate whether the speed of search was equal through the old elements and the new elements, best fitting lines were determined for the functions relating RT to the number of old elements and those relating RT to the number of new elements separately for each participant. Table 1 shows the mean search slopes and intercepts. Generally, search slopes were smaller for target-present trials than for target-absent trials $[F(1,7)=41.53, p<$ $.001]$. For target-present trials there was no difference between the search slope of the function relating RT to the number of old elements and that of the function relating RT to the number of new elements $[F(1,7)=2.16, p>$ $.05]$. For target-absent trials, there was also no difference between the search slope of the function relating RT to the number of old elements and that of the function relating RT to the number of new elements $[F(1,7)=0.07]$. The results with respect to the intercepts of the functions were very similar: Intercepts were smaller for targetpresent than for target-absent trials $[F(1,7)=158.35$, $p<.001]$. For target-present trials there was no difference between the intercept of the function relating RT to the number of old elements and that of the function relating RT to the number of new elements $[F(1,7)=2.23, p>$ $.05]$. For the target-absent trials, there was also no difference between the intercept of the function relating RT to the number of old elements and that of the function relating RT to the number of new elements $[F(1,7)=0.08]$.

Table 3 shows the mean percentages of errors. Overall, participants made $6.2 \%$ errors. Participants more often falsely reported the target to be absent when in fact the target was present than the target to be present when in fact

Target Present

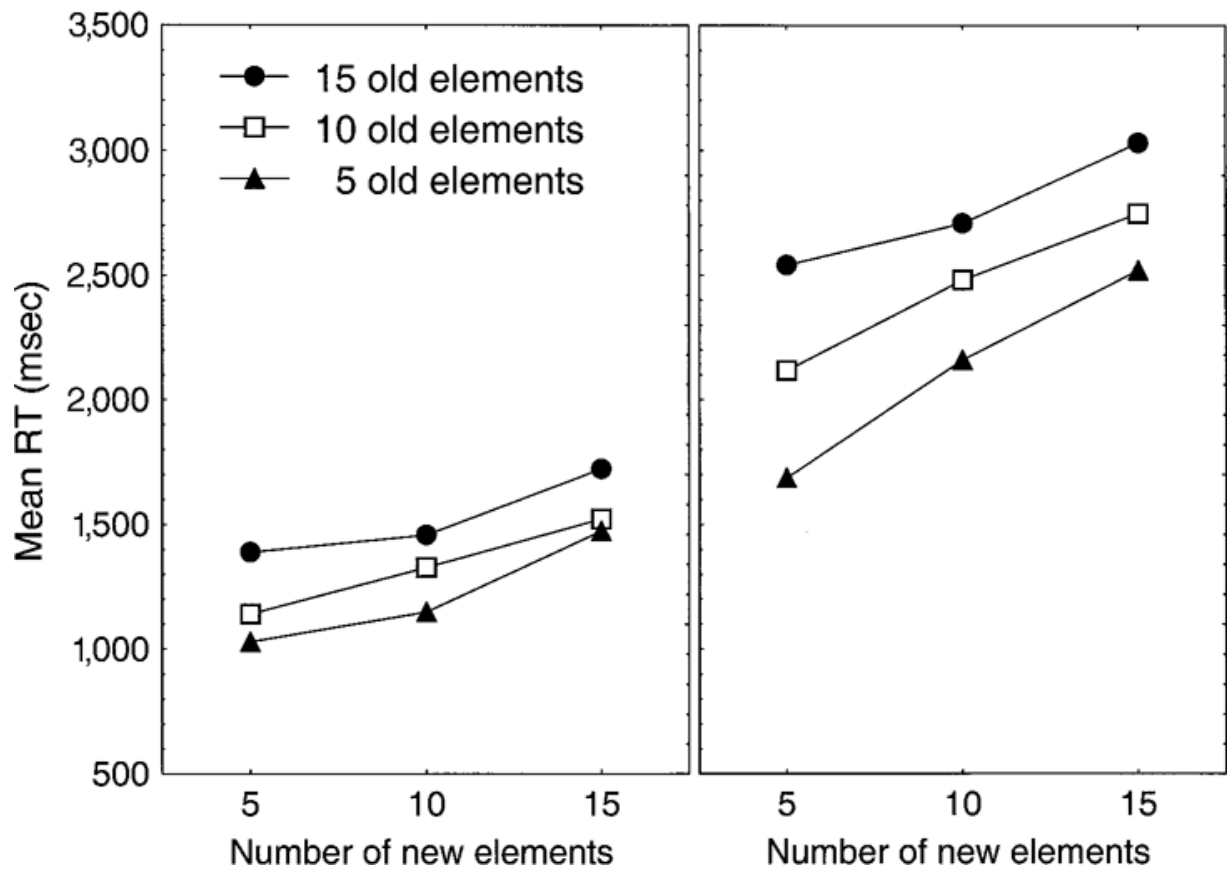

Figure 2. Correct mean reaction time (RT) as a function of the number of new and old elements separately for target-present and target-absent trials in Experiment 2. 
Table 3

Mean Percentages of Errors in Experiment 2

\begin{tabular}{|c|c|c|c|c|c|c|c|c|c|}
\hline & \multicolumn{9}{|c|}{ No. Old Elements } \\
\hline & \multicolumn{3}{|c|}{$\begin{array}{c}5 \\
\text { No. New Elements } \\
\end{array}$} & \multicolumn{3}{|c|}{$\begin{array}{c}10 \\
\text { No. New Elements } \\
\end{array}$} & \multicolumn{3}{|c|}{$\begin{array}{c}15 \\
\text { No. New Element } \\
\end{array}$} \\
\hline & 5 & 10 & 15 & 5 & 10 & 15 & 5 & 10 & 15 \\
\hline Target present & 6 & 11 & 13 & 9 & 13 & 10 & 13 & 14 & 18 \\
\hline Target absent & 1 & 1 & 1 & 0 & 0 & 1 & 2 & 1 & 1 \\
\hline
\end{tabular}

the target was absent $[F(1,7)=24.66, p<.002]$, showing that participants were more inclined to respond "target absent" than "target present." More important was the finding that in line with the RT data, the error rates increased with the number of old elements $[F(2,14)=5.42$, $p<.018]$. Error rates were not affected by the number of new elements $[F(2,14)=1.42, p>.05]$. Generally, error rates were either unaffected or affected in the same direction as RT.

In order to increase the power to detect possible differences between the effects of the number of old elements and those of the number of new elements, the data were combined across Experiments 1 and 2. The results of the analyses of variance (ANOVAs) were basically the same as those of Experiments 1 and 2 separately. Mean RT was larger for the target-absent trials than for the targetpresent trials $[F(1,15)=291.96, p<.001]$.

For the target-present trials, RT increased as a function of the number of old elements $[F(2,30)=23.89, p<$ $.001]$ and as a function of the number of new elements $[F(2,30)=43.94, p<.001]$. There was no interaction between the number of old elements and the number of new elements $[F(4,60)=1.52, p>.05]$.

For the target-absent trials, RT increased as a function of the number of old elements $[F(2,30)=105.87, p<$ $.001]$ and as a function of the number of new elements $[F(2,30)=135.68, p<.001]$. As in the separate analyses, there was a significant interaction between the number of old elements and the number of new elements $[F(4,60)=$ $11.07, p<.001]$, showing that the effects of the number of old and new elements decreased if the total number of elements increased. Again, this finding suggests that observers utilized an upper time limit in finishing the selfterminating search process.

Finally, an ANOVA on the mean search slopes across Experiments 1 and 2 shows that search slopes were smaller for target-present than for target-absent trials $[F(1,15)=$ $88.95, p<.001]$. For target-present trials, there was no difference between the search slope of the function relating RT to the number of old elements and that of the function relating RT to the number of new elements $[F(1,15)=3.53, p>.05]$. For the target-absent trials, there was also no difference between the search slope of the function relating RT to the number of old elements and that of the function relating RT to the number of new elements $[F(1,15)=2.34, p>.05]$. The results with respect to the intercepts of the functions were very similar:
Intercepts were smaller for target-present than for targetabsent trials $[F(1,15)=218.63, p<.001]$. For targetpresent trials, there was no difference between the intercept of the function relating RT to the number of old elements and that of the function relating RT to the number of new elements $[F(1,15)=3.68, p>.05]$. For the target-absent trials, there was also no difference between the intercept of the function relating RT to the number of old elements and that of the function relating RT to the number of new elements $[F(1,15)=2.42, p>.05]$.

\section{Discussion}

The results of Experiment 2 are very similar to those of Experiment 1. Search rates for old elements were identical to those for new elements, indicating that prioritizing did not occur. Apparently, even when old elements were presented with abrupt onsets, participants were not able to inhibit the locations of the old elements to prioritize the selection of new elements. Collapsing the data across Experiments 1 and 2 did not affect the pattern of results. Obviously, participants searched through all elements. The results of Experiments 1 and 2 provide no support for the claim that new elements are prioritized over old elements. To ensure that prioritizing is possible with the present stimuli, a third experiment was executed. In Experiment 3, only the old elements were equiluminant with the background, whereas the new elements were not, yielding an onset of the new elements only.

\section{EXPERIMENT 3}

This experiment replicated Experiment 1 except that only the old elements were equiluminant with the background. The new elements were not equiluminant with the background, yielding an onset of the new elements only.

\section{Method}

Participants. Four male and 4 female participants aged 17 to 24 years took part in the present experiment. Each participant had normal or corrected-to-normal vision.

Apparatus. The apparatus was identical to that used in Experiment 1 .

Task and Stimuli. Task and stimuli were the same as in Experiment 1 except that the luminance of the background was lower upon appearance of the second set of elements (new elements), yielding an onset of the new elements. The luminance of the background was gradually decreased $732 \mathrm{msec}$ after presentation of the old elements (in 15 steps of $32 \mathrm{msec}$ each) until a luminance of $3.8 \mathrm{~cd} / \mathrm{m}^{2}$ during a period of $480 \mathrm{msec}$. Upon presentation of the second set of 
Target Present

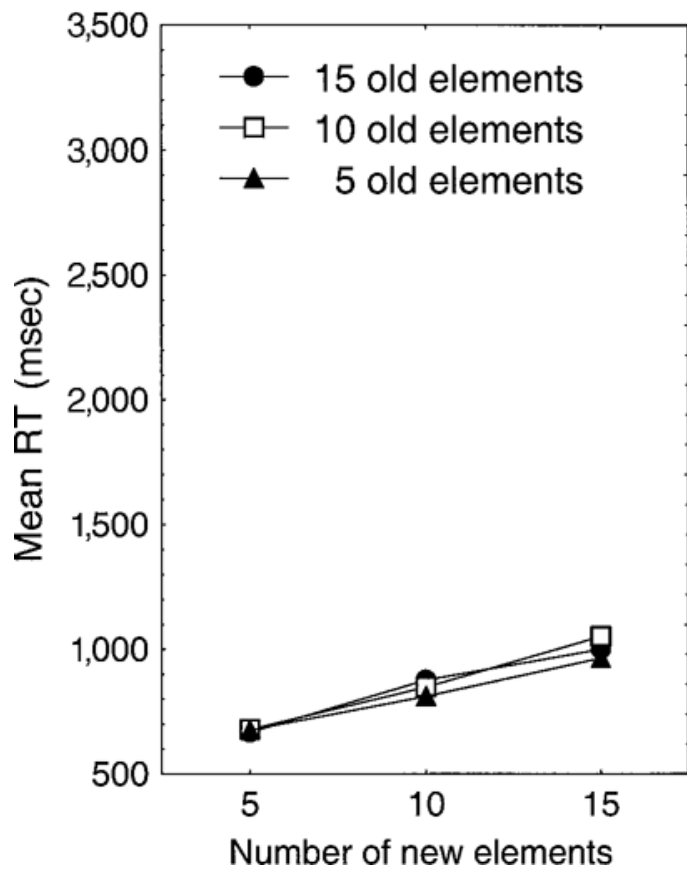

Target Absent

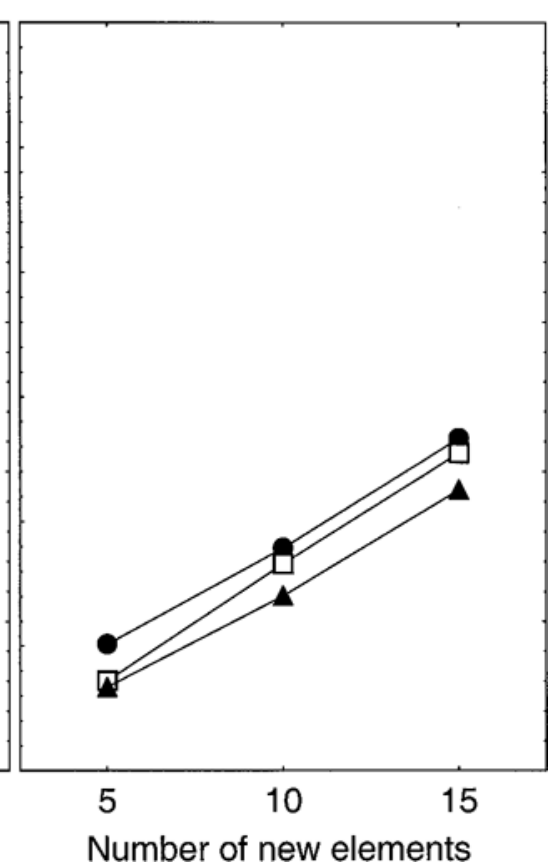

Figure 3. Correct mean reaction time (RT) as a function of the number of new and old elements separately for target-present and target-absent trials in Experiment 3.

elements, 1,244 msec after presentation of the old elements, the background luminance was lower than the luminance of both sets of elements (background: CIE $x, y$ chromaticity coordinates of $.281, .327$, $3.8 \mathrm{~cd} / \mathrm{m}^{2}$; elements: CIE $x, y$ chromaticity coordinates of $.289, .605$, $\left.16.8 \mathrm{~cd} / \mathrm{m}^{2}\right)$.

Design and Procedure. The design and procedure were identical to those used in Experiment 1.

\section{Results}

Figure 3 depicts the mean correct RT as a function of the number of old elements and the number of new elements separately for target-present and target-absent trials. Generally, RT was larger for target-absent trials than for target-present trials $[F(1,7)=14.39, p<.007]$.

For target-present trials, RT increased as a function of the number of new elements $[F(2,14)=15.49, p<.001]$, but not as a function of the number of old elements $[F(2,14)=1.91, p>.05]$. There was no interaction between the number of old elements and the number of new elements $[F(4,28)=1.77, p>.05]$.

For target-absent trials, RT increased as a function of both the number of old elements $[F(2,14)=4.76, p<.027]$ and the number of new elements $[F(2,14)=14.36, p<$ $.001]$. There was no interaction between the number of old elements and the number of new elements $[F(4,28)=$ $1.01, p>.05]$.

To investigate whether the speed of search was equal through the old elements and the new elements, best fitting lines were determined for the functions relating RT to the number of old elements and RT to the number of new elements separately for each participant. Table 1 shows the mean search slopes and intercepts. Generally, search slopes were smaller for target-present trials than for target-absent trials $[F(1,7)=10.19, p<.015]$. For targetpresent trials the search slope of the function relating RT to the number of old elements was significantly smaller than that of the function relating RT to the number of new elements $[F(1,7)=16.24, p<.005]$. This was also true for target-absent trials $[F(1,7)=16.30, p<.005]$. An ANOVA on the intercepts of the functions with target presence (present and absent) and function (the function relating RT to the number of old elements and the function relating RT to the number of new elements) as factors showed a main effect of target presence $[F(1,7)=21.73, p<.002]$ and of function $[F(1,7)=17.13, p<.004]$. There was also a significant interaction between target presence and function $[F(1,7)=11.14, p<.012]$. For target-present trials, the intercept of the function relating RT to the number of old elements was significantly larger than that of the function relating RT to the number of new elements $[F(1,7)=$ $16.17, p<.005]$. This was also true for the target-absent trials $[F(1,7)=16.07, p<.005]$.

Table 4 shows the mean percentages of errors. Overall, participants made $8.2 \%$ errors. Participants more often falsely reported the target to be absent when in fact the target was present than the target to be present when in fact the target was absent $[F(1,7)=10.31, p<.015]$, showing that participants were more inclined to respond "target absent" than "target present." In general, error rates 
Table 4

Mean Percentages of Errors in Experiment 3

\begin{tabular}{|c|c|c|c|c|c|c|c|c|c|}
\hline & \multicolumn{9}{|c|}{ No. Old Elements } \\
\hline & \multicolumn{3}{|c|}{$\begin{array}{c}5 \\
\text { No. New Elements }\end{array}$} & \multicolumn{3}{|c|}{$\begin{array}{c}10 \\
\text { No. New Elements }\end{array}$} & \multicolumn{3}{|c|}{$\begin{array}{c}15 \\
\text { No. New Elements }\end{array}$} \\
\hline & 5 & 10 & 15 & 5 & 10 & 15 & 5 & 10 & 15 \\
\hline Target present & 10 & 10 & 13 & 5 & 18 & 18 & 8 & 16 & 17 \\
\hline Target absent & 2 & 5 & 6 & 1 & 5 & 6 & 3 & 2 & 6 \\
\hline
\end{tabular}

increased with the number of new elements $[F(2,14)=$ $6.83, p<.009]$. Furthermore, error rates were unaffected.

\section{Discussion}

The results of Experiment 3 show that when there was an onset of the new elements, observers prioritized selection of the new elements. Search performance was basically independent of the number of old elements, as evident from the search functions showing that search performance decreased as a function of the number of new elements but not as function of the number of old elements. The results essentially replicate those of Theeuwes et al. (1998) and show again that new elements are prioritized even when they have physically the same color as the old elements.

\section{GENERAL DISCUSSION}

Experiments $1-3$ clearly show that prioritizing selection of new elements is critically dependent on the degree of onset of the new elements. If there is no onset of the new elements (Experiments 1-2), search performance depends on both the number of new elements and the number of old elements. If new elements are presented with abrupt onsets (Experiment 3 ), search performance is independent of the number of old elements, showing that prioritizing occurred. The present study suggests that prioritizing new over old elements is critically dependent on whether the new elements are presented with an abrupt onset upon their appearance.

The present findings are in accordance with a large number of studies showing that abrupt visual onsets receive prioritized selection for processing (e.g., Jonides, 1981; Theeuwes, 1991; Yantis \& Johnson, 1990; Yantis \& Jones, 1991; Yantis \& Jonides, 1984, 1990). Many studies have demonstrated that one single onset in the visual field is generally the first stimulus examined at the expense of other elements in the visual field. For example, Yantis and Jonides (1984) showed that if a target stimulus with an abrupt onset appears among nontargets without an abrupt onset, RT does not increase with the number of elements. Other studies have shown that even in the case of multiple onsets, elements that appear with abrupt luminance increase receive prioritized selection over those without an onset (e.g., Yantis \& Johnson, 1990; Yantis \& Jones, 1991). As such, the present results replicate these earlier findings. However, the finding that prioritizing occurs only when new elements are presented with abrupt onset is problematic for the visual marking account of Watson and Humphreys (1997). According to Watson and Humphreys (1997), prioritized selection of new elements occurs through the inhibition of locations of old elements. As a consequence, old elements do not compete as strongly for selection, resulting in prioritized selection of new elements. Such an account would not predict that prioritizing new over old elements is critically dependent on whether the new elements are presented with an abrupt onset. That is, inhibition of old elements should occur regardless of whether new elements are presented with an abrupt onset.

One might try to save the inhibition account by assuming that prioritizing of new elements by inhibition of old elements can occur only when the new elements are presented with onset. One might, for example, argue that the effect of inhibiting the locations of the old elements is to enable new onsets to enhance activation in an attentional system responsive to dynamic change in the visual environment. If the new elements do not activate this system (i.e., when they are equiluminant with the background), then effective prioritization should not occur. ${ }^{3}$ Although this explanation is in principle viable, it should be noted that it represents a substantial modification of the original idea of visual marking as proposed by Watson and Humphreys (1997). In addition, such an account is not very likely, since there are many studies showing that abrupt visual onsets activate the attentional system directly without any involvement of a top-down inhibition system (e.g., Jonides, 1981; Theeuwes, 1991; Yantis \& Johnson, 1990; Yantis \& Jones, 1991; Yantis \& Jonides, 1984, 1990). Furthermore, it is unusual to assume that an observer is equipped with a voluntary top-down operating system whose effective operation is conditional upon stimulusdriven bottom-up activation of abrupt onsets. Therefore, it seems to be more parsimonious to conclude that prioritized selection of new elements was directly accomplished through the abrupt onsets accompanying their appearance.

There are two features of the present data that appear to be at odds with the results of previous studies. First, the present data show that prioritizing is critically dependent on whether or not the presentation of the new elements was accompanied by a luminance change. These results run counter those of Yantis and Hillstrom (1994), who reported results suggesting that prioritizing new elements over old ones occurs without any luminance change (Experiments 1 and 2) and that if there is a luminance change, prioritizing does not necessarily occur (Experi- 
ment 3). Yantis and Hillstrom concluded that new elements are prioritized by a mechanism that detects the appearance of new perceptual objects instead of a mechanism based on luminance-change detection. As noted by Theeuwes (1995) and, more recently, by Gellatly, Cole, and Blurton (1999), it is questionable whether in Experiment 1 of Yantis and Hillstrom (1994) new objects were indeed equivalent to old objects with respect to luminance. Yantis and Hillstrom used three types of tasks, in which elements were defined by texture, motion, or depth, respectively. Basically, in any of these tasks, the degree of luminance change that occurred upon presentation of the search display was different for the old and new objects. As a result, attentional prioritizing for new objects over old ones might have arisen through the detection of luminance change instead of the detection of the appearance of a new object as such. ${ }^{4}$ In addition, in their Experiment 3, Yantis and Hillstrom presented observers with displays containing old objects only. The objects were revealed by the removal of selected line segments from previously presented figure-eight placeholders. One of the elements was brightened for a 50-msec interval that started when the search display was presented. Yantis and Hillstrom actually found a difference in RT between brightened targets and unbrightened targets. However, they only interpreted the absence of any significant difference in search slopes. As already pointed out by Gellatly et al. (1999), the brightened targets did actually behave like abrupt onset elements in one respect.

Second, the results of Experiment 3 show that RT is independent of the number of old elements even when the number of new elements equals 15 . This suggests that observers can prioritize up to 15 elements simultaneously. The results of previous studies on multiple onsets suggest that there is an upper limit of approximately 4 to the maximum possible number of onset elements that receive priority (Yantis \& Johnson, 1990; Yantis \& Jones, 1991). A possible explanation for this discrepancy might be that in the present study, onsets were never accompanied by offsets, whereas in both Yantis and Johnson and Yantis and Jones, onsets were always accompanied by offsets. That is, upon presentation of the onset stimuli, the no-onset stimuli appeared as a result of the removal of segments of previously presented figureeight placeholders. Furthermore, the presentation of the elements with onsets was often accompanied by the complete offset of several figure-eight placeholder figures. As noted, Theeuwes (1991) showed that offsets can be just as powerful as onsets in receiving priority for selection. It is therefore likely that there are no processing limitations of the attentional priority system, as suggested by Yantis and Johnson (1990) and Yantis and Jones (1991). Instead, in their study, apart from the onset stimuli, the offset stimuli might have also received attentional priority (see also Martin-Emerson \& Kramer, 1997; Miller, 1989). How many elements can receive attentional priority through abrupt onset is an open question.
At this point, it should be noted that the experiments reported in the present paper do not allow any conclusion with respect to whether prioritized selection through abrupt onsets is a bottom-up stimulus-driven process or not. Obviously, observers knew that the target they were looking for could occur only among the second set of elements. As a consequence, both bottom-up and top-down control of visual attention would have led to prioritized selection of the abrupt onsets in Experiment 3.

In sum, the present results suggest that prioritizing selection of new elements in a multiple-element display is mediated by a mechanism that is critically dependent on the detection of luminance changes. The present study demonstrates that observers are able to prioritize up to 15 elements simultaneously. Since prioritizing selection of a subset of elements is an important phenomenon, future research should further examine the possible mechanisms by which this occurs.

\section{REFERENCES}

Gellatly, A., Cole, G., \& Blurton, A. (1999). Do equiluminant object onsets capture visual attention? Journal of Experimental Psychology: Human Perception \& Performance, 25, 1609-1624.

Ives, H. E. (1912). Studies of the photometry of different colours: I. Spectral luminosity curves obtained by equality of brightness photometer and the flicker photometer under similar conditions. Philosophical Magazine, 24, 149-188.

JONIDES, J. (1981). Voluntary versus automatic control over the mind's eye's movement. In J. Long \& A. Baddeley (Eds.), Attention and performance IX (pp. 187-203). Hillsdale, NJ: Erlbaum.

Martin-Emerson, R., \& Kramer, A. F. (1997). Offset transients modulate attentional capture by sudden onsets. Perception \& Psychophysics, 59, 739-751.

Miller, J. (1989). The control of attention by abrupt visual onsets and offsets. Perception \& Psychophysics, 45, 567-571.

Theeuwes, J. (1991). Exogenous and endogenous control of attention: The effect of visual onsets and offsets. Perception \& Psychophysics, 49, 83-90.

Theeuwes, J. (1995). Abrupt luminance change pops out; abrupt color change does not. Perception \& Psychophysics, 57, 637-644.

Theeuwes, J., Kramer, A. F., \& Atchley, P. (1998). Visual marking of old objects. Psychonomic Bulletin \& Review, 5, 130-134.

Watson, D. G., \& Humphreys, G. W. (1997). Visual marking: Prioritizing selection for new objects by top-down attentional inhibition of old objects. Psychological Review, 104, 90-122.

Watson, D. G., \& Humphreys, G. W. (1998). Visual marking of moving objects: A role for top-down feature-based inhibition in selection. Journal of Experimental Psychology: Human Perception \& Performance, 24, 946-962.

YANTIS, S., \& Hillstrom, A. P. (1994). Stimulus-driven attentional capture: Evidence from equiluminant visual objects. Journal of Experimental Psychology: Human Perception \& Performance, 20, 95-107.

YANTIS, S., \& Johnson, J. C. (1990). On the locus of visual selection: Evidence from focused attention tasks. Journal of Experimental Psychology: Human Perception \& Performance, 16, 135-149.

YANTIS, S., \& JonEs, E. (1991). Mechanisms of attentional selection: Temporally modulated priority tags. Perception \& Psychophysics, 50, 166-178.

YANTIS, S., \& JonidES, J. (1984). Abrupt visual onsets and selective attention: Evidence from visual search. Journal of Experimental Psychology: Human Perception \& Performance, 10, 601-621.

YANTIS, S., \& JonidES, J. (1990). Abrupt visual onsets and selective attention: Voluntary versus automatic allocation. Journal of Experimental Psychology: Human Perception \& Performance, 16, 121-134. 


\section{NOTES}

1. We use the term onset to refer to the presentation accompanied by a luminance increment and the term offset to the presentation accompanied by a luminance decrement.

2. Due to retinal inhomogeneities, deviance from isoluminance may occur in the periphery. However, such an effect would go against a hypothesis that states that prioritized selection of new elements is due to luminance change.
3. This possibility was suggested by one of the reviewers.

4. In the Yantis and Hillstrom (1994) study, observers were presented with displays containing only a few elements that were arranged on an imaginary circle around central fixation. This low number of elements arranged in a highly structured display could have led observers to note a difference in the global appearance of the display based on a comparison between the old and new elements. As a consequence, new objects might have simply been detected faster as a result of a direct comparison between both displays (see also Theeuwes, 1995, for a similar argument).

(Manuscript received December 31, 1999;

revision accepted for publication September 8, 2000.) 